\title{
Age estimation in children by measurement of open apices in teeth: a European formula
}

\author{
Roberto Cameriere • Danilo De Angelis • \\ Luigi Ferrante $\cdot$ Francesco Scarpino • \\ Mariano Cingolani
}

Received: 18 January 2007 / Accepted: 18 April 2007

(C) Springer-Verlag 2007

\begin{abstract}
The aim of the present paper was to improve and expand research with a larger number of children from various European countries and to provide a common formula useful for all these countries. Orthopantomographs taken from 2,652 European Caucasian children (1,382 boys, 1,270 girls) aged between 4 and 16 years were analyzed. The children came from Croatia, Germany, Kosovo, Italy, Slovenia, Spain, and the UK. Following the pilot study, subjects' age was modeled as a function of gender $(g)$, morphological variables (predictors) $\times 5$ (second premolar), $s$ (sum of normalized open apices) $N_{0}$, and the first-order interaction between $s$ and $N_{0}$. The results showed that all these variables contributed significantly to the fit, so that all were included in the regression model, yielding the following linear regression formula: Age $=8.387+$ $0.282 g-1.692 \times 5+0.835 N_{0}-0.116 s-0.139 s \times N_{0}$, where $g$ is a variable, 1 for males and 0 for females. The equation
\end{abstract}

R. Cameriere $(\bowtie) \cdot$ F. Scarpino $\cdot$ M. Cingolani

Institute of Legal Medicine, University of Macerata,

Via Don Minzoni 9,

62100 Macerata, Italy

e-mail: r.cameriere@unimc.it

D. De Angelis

Laboratorio di Antropologia e Odontologia forense (LABANOF), Institute of Legal Medicine, University of Milano,

Via Mangiagalli 37,

20133 Milan, Italy

\section{Ferrante}

Institute of Microbiology and Biomedical Sciences, Polytechnic University of Marche, Ancona,

Via Ranieri 65,

60100 Ancona, Italy

R. Cameriere

Institute of Forensic Medicine, University of Macerata,

Via Don Minzoni 9,

62100 Macerata, Italy explained $86.1 \%\left(R^{2}=0.861\right)$ of total deviance. The median of the residuals ( $=$ observed age minus predicted age) was -0.114 years, with (RefB.2) interquartile range $=1.22$ years.

Keywords Forensic science - Age estimation - Open apices · Mineralization $\cdot$ Multiple regression

\section{Introduction}

Evaluation of skeletal age using radiological techniques is a suitable criterion for assessing individual biological maturation and is normally applied to answer forensic, pediatric, and orthodontic questions. Over the last century, social problems and the discovery of X-rays encouraged the study of a number of methods for age evaluation in both adults and non-adults.

In non-adult subjects, dental age is one of the major indicators of maturity and was used in the UK to estimate age in children before it became obligatory to register births in 1837. Saunders, a dentist, wrote a paper entitled "The teeth: a test of age, considered with reference to the factory children," which was addressed to the members of both Houses of Parliament [1].

After the discovery of X-rays, several methods for age estimation were studied. At present, the need to estimate the age of living individuals is a problem of increasing interest in our community, due to the progressively increasing numbers of persons without legal documentation of birth, who are suspected of having committed crimes and for whom it is necessary to assess actual age to establish imputability.

Teeth are the most frequently used part of the body analyzed for age estimation. The high number of teeth and the continuous modification of both crown and root in 
children mean that several methods of estimating age from teeth can be applied.

The routine use of X-rays since 1950 induced researchers to focus on mineralization. In particular, in 1973 Demirjian et al. [2] studied one method of age estimation. Their original sample comprised 1,446 boys and 1,482 girls of French-Canadian origin, and their data were later compared with other sample groups from several nationalities. Most of the results revealed the fact that the standards of dental maturation described by Demirjian et al. are not always suitable for these countries [3-7]. A few papers modified the original regression model with new samples [e.g., 8, 9], one of which assessed a large sample from eight countries worldwide [10]. Although these studies validated the method, they also highlighted the need to apply a particular regression model to each country.

In 2006, Cameriere et al. [11] presented a method for assessing chronological age in children based on the relationship between age and measurement of open apices in teeth, which gave reliable estimates of the ages of 455 Italian Caucasian children. In the same year, the same authors also published a paper with additional samples from Kosovo and Slovenia, for a total number of 1,100 children [12]. Its aim was to improve and expand research with new numbers of European children from various countries and to complete a common formula useful for all of them.

\section{Materials and methods}

Orthopantomographs from 2,652 European Caucasian healthy children (1,382 boys, 1,270 girls) aged between 4 and 16 years were analyzed (Table 1). The children came from Croatia, Germany, Kosovo, Italy, Slovenia, Spain, and the UK (Table 2). The orthopantomographs were taken as part of the routine treatment between 2000 and 2006.

Table 1 Age and sex distribution of studied individuals

\begin{tabular}{lrrr}
\hline Age & Females & Males & Total \\
\hline 4 & 14 & 15 & 29 \\
5 & 31 & 55 & 86 \\
6 & 72 & 77 & 149 \\
7 & 113 & 117 & 230 \\
8 & 161 & 161 & 322 \\
9 & 178 & 174 & 352 \\
10 & 168 & 180 & 348 \\
11 & 156 & 154 & 310 \\
12 & 123 & 153 & 276 \\
13 & 125 & 137 & 262 \\
14 & 76 & 90 & 166 \\
15 & 32 & 40 & 72 \\
16 & 21 & 29 & 50 \\
Total & 1,270 & 1,382 & 2,652 \\
\hline
\end{tabular}

Table 2 Distribution of studied individuals in various countries

\begin{tabular}{lccc}
\hline Country & Females & Males & Total \\
\hline Croatia & 120 & 148 & 268 \\
Germany & 251 & 249 & 500 \\
Kosovo & 162 & 164 & 326 \\
Italy & 232 & 241 & 473 \\
Slovenia & 147 & 160 & 307 \\
Spain & 208 & 254 & 462 \\
UK & 150 & 166 & 316 \\
Total & 1,270 & 1,383 & 2,652 \\
\hline
\end{tabular}

Orthopantomographs were made X-rays that were unclear or which showed hypodontia, gross pathology, or previous orthodontic treatment were excluded. The chronological age of each subject was calculated by subtracting the date of the $\mathrm{X}$-ray from the date of birth, after converting both to a decimal age by the method of Eveleth and Tanner [13].

$\mathrm{X}$-rays were in digital form or were digitalized on a scanner, and images were recorded on computer files, which were processed by a computer-aided drafting program (Adobe Photoshop 7). The method is fully explained in Cameriere et al. [11]. Briefly, the left permanent mandibular teeth, except the wisdom tooth, were assessed, with the apical ends of the roots completely closed $\left(N_{0}\right)$ and were ascertained. Teeth with incomplete root development, and therefore with open apices, were also examined. For teeth with one root, the distance $A_{i}, i=1, \ldots, 5$, between the inner side of the open apex was measured. For example, $A_{1}$ denotes the distance between the inner side of the open apex of the first incisor. For teeth with two roots, $A_{i}, i=6,7$, the sum of the distances between the inner sides of the two open apices was evaluated. To take into account the effect of possible differences in magnification and angulation among X-rays, measurements were normalized by dividing by tooth length $\left(L_{i}, i=1, \ldots, 7\right)$. Lastly, dental maturity was evaluated using the normalized measurements of the seven permanent left mandibular teeth $\left(x_{i}=A_{i} / L_{i}, i=1, \ldots, 7\right)$, the sum of normalized open apices $\left(s=x_{1}+x_{2}+x_{3}+x_{4}+x_{5}+x_{6}+\right.$ $\left.x_{7}\right)$, and the number $\left(N_{0}\right)$ of teeth with root development completed.

Measurements were carried out by four different observers. Assessment of intra-observer and inter-observer reproducibility was checked on an independent sample of 40 panoramic radiographs.

\section{Statistical analysis}

All the morphological variables, $x_{i}, i=1, \ldots, 7, s$, and $N_{0}$, and subjects' gender were entered in an Excel file, to be used as predictive variables for age estimation in subsequent 
Table 3 Correlation coefficients between age and morphological variables

\begin{tabular}{lllllllll}
\hline$x_{7}$ & $x_{6}$ & $x_{5}$ & $x_{4}$ & $x_{3}$ & $x_{2}$ & $x_{1}$ & $N_{0}$ & $S$ \\
\hline-0.83 & -0.61 & -0.80 & -0.84 & -0.80 & -0.60 & -0.49 & 0.91 & -0.86
\end{tabular}

statistical analysis. Chronological age, calculated by subtracting the date of birth from the date of the radiograph, was also recorded.

Intra-observer and inter-observer reproducibility of measurement was assessed by the concordance correlation coefficient. Correlation coefficients between age and predictive variables were also calculated. To obtain an estimate of age as a function of the morphological variables and subjects' gender and nationality, a multiple linear regression model with first-order interactions was developed by selecting those variables that contributed significantly to age estimations using the stepwise selection method. Analysis of covariance was then applied to study possible interactions between significant morphological variables and gender. Statistical analysis was performed with S-PLUS 6 statistical programs (S-PLUS 6.1 for Windows, Professional Edition, Release 1). The significance threshold was set at $5 \%$.

\section{Results}

There were no statistically significant inter-observer and intraobserver differences between the paired sets of measurements carried out on the reexamined panoramic radiographs. Pearson's correlation coefficients between age and morphological variables showed that all of them were significantly correlated with age (Table 3).

Following the pilot study [11], subjects' age was modeled as a function of gender $(g)$, morphological

Table 4 Stepwise regression analysis predicting chronological age from chosen predictors

\begin{tabular}{lrlrl}
\hline & Value & Std. Error & $t$ value & $P$ \\
\hline Intercept & 8.387 & 0.078 & 107.6 & $<0.001$ \\
$g$ & 0.282 & 0.039 & 7.3 & $<0.001$ \\
$x 5$ & -1.692 & 0.118 & -14.3 & $<0.001$ \\
$N_{0}$ & 0.835 & 0.014 & 61.3 & $<0.001$ \\
$s$ & -0.116 & 0.013 & -8.9 & $<0.001$ \\
$s \times N_{0}$ & -0.139 & 0.012 & -11.7 & $<0.001$ \\
\hline
\end{tabular}

$s / N_{0}$ interaction between variables $s$ and $N_{0}$ variables (predictors) $\times 5$ (second premolar), $s, N_{0}$, and the first-order interaction between $s$ and $N_{0}$. The results (Table 4) show that all the considered variables contributed significantly to the fit, so that all of them were included in the regression model, yielding the following linear regression formula:

$$
\begin{aligned}
\text { Age }= & 8.387+0.282 g-1.692 \times 5+0.835 N_{0} \\
& -0.116 s-0.139 s \cdot N_{0}
\end{aligned}
$$

where $g$ is a variable, 1 for males and 0 for females.

When nationality was added to the predictor variables, Akaike information criterion (AIC) of the statistical model increased from $\mathrm{AIC}=2,185$ to $\mathrm{AIC}=2,211$, and the fit had a modest and not significant improvement from $R^{2}=0.904$ to $R^{2}=0.905$. These results indicated that nationality did not contribute significantly to age estimations, and thus, it was excluded from the set of predictor variables.

In Eq. 1, only the intercept varies with gender, and therefore, sexual dimorphism does not change with age, but the equation does indicate earlier dental maturity for girls at all ages.

Equation 1, with the considered variables, explained $86.1 \%\left(R^{2}=0.861\right)$ of total deviance. The median of the residuals ( $=$ observed age minus predicted age) was -0.114 years, with interquartile range, $\mathrm{IQR}=1.22$ years.

The residual plot (Fig. 1, right panel) shows no obvious pattern. The observed versus predicted plot (Fig. 1, left panel) shows that the regression model fits the trend of the data reasonably well. Hence, both diagnostic plots support our chosen model.

\section{Discussion}

Several parties of body [14-16] and, in particular, the teeth are often used as age indicators in both biological and forensic issues [17, 18]. In 2006, Cameriere et al. [11] published a new method for age estimation using measurement of open apices in teeth. In the original paper, an Italian sample aged between 5 and 15 years was studied.

The results showed that gender and variables $x_{5}$ (second premolar), $s, N_{0}$, and the first-order interaction between $s$ and $N_{0}$ contributed significantly to the fit, so that only these 
Fig. 1 Plots of residuals against fitted values (right panel) and of observed against predicted values (left panel) using regression model 1

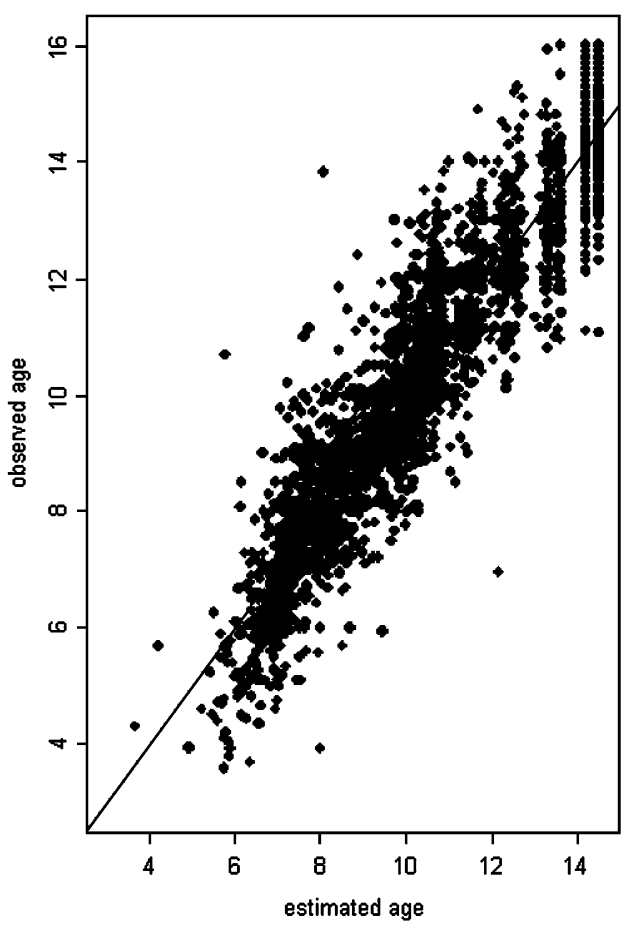

variables were included in the regression model, yielding the following linear regression formula:

$$
\begin{aligned}
\text { Age }= & 8.971+0.375 g+1.631 x_{5}+0.674 N_{0} \\
& -1.034 s-0.176 s \times N_{0}
\end{aligned}
$$

Statistical analysis indicated that these morphological variables explain $83.6 \%$ of variations in estimated chronological age. The median of the absolute value of residual errors between actual and estimated ages was 0.035 years (interquartile range $=1.18$ years).

Larger samples from Kosovo and Slovenia were later studied. Also in this case, the similar variables contributed significantly to the fit, yielding the following linear regression formula:

$$
\begin{aligned}
\text { Age }= & 9.063+0.386 g+1.268 x_{5}+0.676 N_{0} \\
& -0.913 s-0.175 s \times N_{0}
\end{aligned}
$$

These morphological variables explain $83.3 \%$ of variance, and the median of the absolute value of residual error was less than 0.03 years. Therefore, the addition of new samples from other countries did not modify the parameters to any significant extent.

In the present paper, data from 2,652 European Caucasian children between 4 and 16 yielded the linear regression formula 1.

In this case, morphological variables explain $86.2 \%$ of variance, and the median of the absolute value of residual error is less than 1 year. As the results did not show any statistically significant difference between countries, one regression equation could be applied to all samples.

Data from children of the European countries added in this study increased the area to which the regression formula can be applied. The heterogeneity of the sample also suggested the possibility of using Eq. 1 for all Caucasian people, as there are no anthropological reasons for believing that it is necessary to use a different equation for European countries not included in this study. However, as soon as possible, the regression model will be compared with data from other European countries. In non-European countries, where anthropological reasons and nutritional status suggest careful study of several sample populations of Africa, America, and Asia, different issues arise.

Equation 1 will be also shown on website http:// agestimation.unimc.it. If research on samples of different nationalities requires new parameter estimations, they will be re-evaluated to find a new equation with a more general validity. If that is the case, the countries involved in the estimation procedure will be mentioned on the same website to indicate clearly in which countries the proposed formula can be used.

Future research should aim at using our European sample to compare the reliability of our method with other methods for age estimations as, in particular, Willems, Demirjian, Nolla, and Haavikko [2, 19, 20].

Acknowledgements The authors would like to thank the Bejtulla Zeqiri Department of Orthodontics, University of Prishtina, Kosovo; José L. Prieto, Laboratorio de Antropología y Odontología Forense, 
Instituto Anatómico Forense, Madrid, Spain; Hrvoje Brkic, School of Dental Medicine, Department of Dental Anthropology, University of Zagreb, Croatia; Brita Willershausen and Ioannis Moschos, Poliklinik für Zahnerhaltungskunde, Klinikum der Johannes Gutenberg, Universität Mainz, Germany; Branko Eremenc, Institute of Forensic Medicine, Faculty of Medicine, University of Ljubljana, Slovenia; and Helen M. Liversidge, Dental Institute, Bart's and The London School of Medicine and Dentistry, London, UK, who kindly supplied sample data from their respective countries.

\section{References}

1. Saunders E (1837) The teeth: a test of age considered with reference to the factory children. Renshaw Strand, London

2. Demirjian A, Goldstein H, Tanner JH (1973) A new system of dental age assessment. Hum Biol 45:221-227

3. Koshy S, Tandon S (1998) Dental age assessment: the applicability of Demirjian's method in south Indian children. Forensic Sci Int 94:73-85

4. Nykanen R, Espeland L, Kvaal SI, Krogstad O (1998) Validity of the Demirjian method for dental age estimation when applied to Norwegian children. Acta Odontol Scand 56:238-244

5. Liversidge HM, Speechly T, Hector MP (1999) Dental maturation in British children: are Demirjian's standards applicable? Int J Paediatr Dent 9:263-269

6. Prabhakar AR, Panda AK, Raju OS (2002) Applicability of Demirjian's method of age assessment in children of Davangere. J Indian Soc Pedod Prev Dent 20:54-62

7. Eid RM, Simi R, Friggi MN, Fisberg M (2002) Assessment of dental maturity of Brazilian children aged 6 to 14 years using Demirjian's method. Int J Paediatr Dent 12:423-428

8. Chaillet N, Willems G, Demirjian A (2004) Dental maturity in Belgian children using Demirjian's method and polynomial functions: new standard curves for forensic and clinical use. J Forensic Odonto Stomatol 22:18-27
9. Chaillet N, Demirjian A (2004) Dental maturity in south France: a comparison between Demirjian's method and polynomial functions. J Forensic Sci 49:1059-1066

10. Chaillet N, Nystrom M, Demirjian A (2005) Comparison of dental maturity in children of different ethnic origins: international maturity curves for clinicians. J Forensic Sci 50:1164-1174

11. Cameriere R, Ferrante L, Cingolani M (2006) Age estimation in children by measurement of open apices in teeth. Int J Legal Med 120:49-52

12. Cameriere R, Ferrante L, Scarpino F, Branko E, Betjtulla Z (2006) Dental age estimation of growing children: comparison among various European countries. Acta Stomatol Croat 40:255-262

13. Eveleth PB, Tanner JM (1990) Worldwide variation in human growth, edn. Cambridge Univ. Press, Cambridge, pp 6-7

14. Mühler M, Schulz R, Schmidt S, Schmeling A, Reisinger W (2006) The influence of slice thickness on assessment of clavicle ossification in forensic age diagnostics. Int J Legal Med 120:15-17

15. Schmeling A, Baumann U, Schmidt S, Wernecke KD, Reisinger W (2006) Reference data for the Thiemann-Nitz method of assessing skeletal age for the purpose of forensic age estimation. Int J Legal Med 120:1-4

16. Cameriere R, Ferrante L, Mirtella D, Cingolani M (2006) Carpals and epiphyses of radius and ulna as age indicators. Int $\mathrm{J}$ Legal Med 120:143-146

17. Olze A, Bilang D, Schmidt S, Wernecke KD, Geserick G, Schmeling A (2005) Validation of common classification systems for assessing the mineralization of third molars. Int J Legal Med 119:22-26

18. Paewinsky E, Pfeiffer H, Brinkmann B (2005) Quantification of secondary dentine formation from orthopantomograms - a contribution to forensic age estimation methods in adults. Int $\mathrm{J}$ Legal Med 119:27-30

19. Nolla CM (1960) The development of the permanent teeth. J Dent Child 27:254-266

20. Haavikko K (1974) Tooth formation age estimated on a few selected teeth. A simple method for clinical use. Proc Finn Dent Soc 70:15-19 